\title{
Ground-State Roughness of the Disordered Substrate and Flux Lines in $d=2$
}

\author{
Chen Zeng $^{a}$, A. Alan Middleton ${ }^{a}$ and Y. Shapir ${ }^{b}$ \\ ${ }^{a}$ Department of Physics, Syracuse University, Syracuse, NY 13210, USA \\ ${ }^{b}$ Department of Physics and Astronomy, University of Rochester, Rochester, NY 14627, USA
} (Submitted 28 May 1996 - to appear in PRL)

\begin{abstract}
We apply optimization algorithms to the problem of finding ground states for crystalline surfaces and flux lines arrays in presence of disorder. The algorithms provide ground states in polynomial time, which provides for a more precise study of the interface widths than from Monte Carlo simulations at finite temperature. Using $d=2$ systems up to size $420^{2}$, with a minimum of $2 \times 10^{3}$ realizations at each size, we find very strong evidence for a $\ln ^{2}(L)$ super-rough state at low temperatures.
\end{abstract}

Typeset using REVTEX 
The flux-lines array formed in a 2D type-II dirty superconductor with the magnetic field parallel to the plane and the surface configurations of a crystalline defect-free material deposited on a disordered substrate (DS) are closely related systems. They have been studied both for the intrinsic interest and because they serve as prototypical models for elastic media in a disordered environment. Both have low temperature glassy phases in which equilibrium and dynamic properties are dominated by the disorder. In the continuum limit they are both described by the random-phase sine-Gordon model (RSGM). However, analytic attempts at understanding the equilibrium properties of the glassy phase based on the RSGM have yielded conflicting results [1]2]. Finite temperature simulations of the RSGM or the corresponding discrete Gaussian model for the disordered substrate have also been ambiguous [3 $[$ ]. Moreover, it is not clear to which extent universality arguments, which yield the RSGM as the continuum limit, can be trusted at temperatures well below the glass transition.

The aim of the present work is to address both issues by finding the exact minimum energy configurations in discrete models of the DS surface and that of the flux-lines array. This yields their $\mathrm{T}=0$ shapes for any given disorder realization; averaging over disorder allows for the evaluation of their averaged physical properties. In particular, the quantities that theory and simulation has focused on are the height-height correlations in the disordered substrate model and the line-line correlations in the flux-line model. The flux-lines system is discretized by its mapping to a surface model (see below) and one may observe the transition by inspecting the roughness scaling of the respective surfaces instead. The transition into the glassy phase is exhibited by a change in these height-height correlations in both models.

The predictions from the analytic studies of the RSGM are as follows: Above the transition temperature $T=T_{g}$ the surface is always logarithmically rough, with a prefactor proportional to T. Below the transition temperature, renormalization group (RG) calculations [1] predict a $\ln ^{2}(L)$ behavior, while variational approaches [2] predict the persistence of the $\ln (L)$ behavior but with the prefactor unchanging for $T \leq T_{g}$.

Numerical simulations have differed in their results as well. Simulations of the RSGM 
with weak coupling have shown no transition at all [3]. Others have shown a transition with a $\ln (L)$ [4] or a $\ln ^{2}(L)$ behavior [5]. MC simulations of the discrete Gaussian version of the model have exhibited the transition but the behavior of the roughness could fit better the $\ln (L)$ [6] or the $\ln ^{2}(L)$ [7], depending on the way the data was interpreted.

As shown below we find very strong evidence that the ground states configurations for both systems exhibit a $\ln ^{2}(L)$ roughness. This reinforces the RG predictions and, moreover, indicates that they hold all the way to $\mathrm{T}=0$, a much wider temperature range than might be anticipated. We are able to make strong conclusions since we are able to calculate averages over many more realizations and at larger length scales. As we show below, studying the correlation function in wavevector space also allows for a clearer distinction between the two predictions.

To be specific, the ground-state properties of two interface models with quenched random impurities are investigated in this letter. The first model, i.e., the disordered-substrate surface model [6], is described by height variables $h_{i}$ defined on every site $i$ of a square lattice. The coupling between height variables is governed by the following Hamiltonian:

$$
H_{1}=\kappa \sum_{<i, j>}\left|h_{i}-h_{j}\right|
$$

where $\kappa$ is the step energy, and the summation runs over all nearest-neighbor bonds of the square lattice. (The discrete Gaussian model usually replaces this Hamiltonian near and above $T_{c}$ where they belong to the same universality class). In the case of a flat substrate the $h_{i}$ takes integer values. To model the disordered substrate, however, the height $h_{i}$ takes the values $h_{i}=n_{i}+d_{i}$ where $n_{i}$ is an integer and the quenched random height $d_{i}$ is chosen uniformly (and independently for each site) in the interval $(-1 / 2,+1 / 2]$. Clearly, any surface configuration can be viewed as an interface along (001)-direction of a simple cubic lattice with the substrate being identified with the basal plane.

The second model is the Triangular Ising Solid-on-Solid model (TISOS) [8] where the surface configuration is described by height variables $h_{i}$ which are defined on every site $i$ of a triangular lattice and take only integer values. The Hamiltonian is chosen to be of the 
form:

$$
H_{2}=\sum_{<i j>} J_{i j}\left(\left|h_{i}-h_{j}\right|-1\right)
$$

where the summation again runs over all nearest-neighbor bonds, and the coupling constants $J_{i j}$ are chosen uniformly (and independently for each bond) in the interval $[0,1]$ to simulate the quenched point disorder. In contrast to the first model, further constraints are imposed on the height variables: (1) the height difference $\left|h_{i}-h_{j}\right|$ for every bond must be either 1 or 2, and (2) the total height increment (clockwise or counterclockwise) along any elementary triangle is zero. See Fig. 1 for an example of this construction. It is straightforward to check that the SOS surface so defined describes an interface along the (111)-direction of a simple cubic lattice.

There exists yet another well-known representation of the TISOS model [8], i.e., the dimer-covering model on a hexagonal lattice, which not only makes transparent the equivalence of the TISOS to an array of self-avoiding fluxlines in $d=2$ dimensions but also facilitates the implementation of an efficient network optimization algorithm to study the ground-state properties of the TISOS model. Here each bond on the triangular lattice with $\left|h_{i}-h_{j}\right|=2$ is identified with a dimer on the dual bond of the hexagonal lattice. See Fig. 1 for a diagram of this construction. A simple inspection shows that a surface configuration $\left\{h_{i}\right\}$ is mapped onto a complete dimer covering of the hexagonal lattice. The fluxline representation results from the transition graph (superposition of the two dimer-covering configurations) between an arbitrary dimer-covering and a fixed reference dimer-covering where all vertical bonds of the hexagonal lattice are covered with dimers. Here the vertical direction then specifies the direction of the fluxlines (or the direction of an external magnetic field). See Fig. 1 for an explicit illustration. Let $E_{r}, E_{d}$ and $E_{f}$ denote the sum of the energies of all vertical bonds, of all bonds that are covered by dimers, and of all bonds that coincide with the fluxlines, respectively. The following equality then holds: $E_{d}\left(\left\{J_{i j}\right\}\right)-E_{r}\left(\left\{J_{i j}\right\}\right)=E_{f}\left(\left\{K_{i j}\right\}\right)-n L$, where $n$ signifies the number of fluxlines, $L$ is the length of the system (see Fig. 1), and $K_{i j}$ denotes a transformed set of random bonds 
according to $K_{i j}=J_{i j}$ for all non-vertical bonds, and $K_{i j}=1-J_{i j}$ for all vertical bonds. This simple exercise therefore demonstrates that all different representations (interface along (111)-direction, dimers, fluxlines) are also energetically equivalent with a suitable transformation of the random bonds that still maintains its uniform and independent distribution.

For the purpose of comparison, we now briefly summarize the known results on the ground-state properties of the above two models in the absence of disorder. In the DS model, the surface is trivially flat (constant $h_{i}$ ). In the latter case (TISOS), however, the ground state is highly degenerate with a macroscopic entropy per lattice site [10]. By exploring the equivalence between the ground-state ensemble of TISOS and that of the antiferromagnetic Ising model on a triangular lattice (thus the name TISOS), Blöte and Hilhorst [9] have shown that the ground state is logarithmically rough due to the entropic fluctuations. The structure factor $S(k)=\left\langle|h(\vec{k})|^{2}\right\rangle$ in the long wavelength limit is given by $S(k)=\left(K_{s} \vec{k}^{2}\right)^{-1}$, with the stiffness constant $K_{s}=\pi / 9$. The width $W$ of the surface then satisfies $W^{2} \equiv\left\{h^{2}(\vec{r})\right\}-\{h(\vec{r})\}^{2}=\left(\pi K_{s}\right)^{-1} \ln (L)+\cdots$ for $L \rightarrow \infty$, where $L$ is the linear size of the system. Here we have used $\{\cdot\}$ for the average over lattice sites, and $\langle\cdot\rangle$ for the ground-state ensemble average.

The problem of finding the ground states of the above models in the presence of disorder is that of minimizing the total energy given by Eq. (1) and Eq. (2) respectively. Different representations of the models naturally lend themselves to various efficient network optimization algorithms, of which we employed the network maximum-flow algorithm and the minimum-cost perfect matching algorithm $\| 11$ by utilizing the interface and dimer covering representations respectively.

In the case of the interface representation, we apply the algorithm presented in [12]. For a directed graph with given "edge capacities" indicating the maximum amount of fluid that can flow from one node to another along the directed edge, the maximum flow algorithm determines the maximum amount of flow that can be sustained between two given nodes, the source and the sink, given flow conservation at all other nodes. It is easily shown that the maximum flow value is identical to the value of the minimum "cut", which is defined as 
the weight of the set of edges with minimum total capacities that, when cut, disconnect the source and the sink [11]. This comes about because the minimum cut is the "bottleneck" through which all flow must pass. To apply this algorithm, a finite 3-dimensional graph is constructed whose minimum cut directly corresponds to the minimum energy interface in the height model. For the DS and TISOS models, graphs can be constructed whose minimum cut surfaces lack overhangs and minimize the appropriate energies. For example, the graphs for the TISOS model have the same topology as for the random-bond Ising model (RBIM) as described in [12], but the disorder is periodic in the (111) direction (in RBIM, the disorder is uncorrelated). Once the corresponding graph is constructed, the max-flow/mincut algorithm can be directly applied. Specifically, the push-relabel algorithm and code developed by Cherkassky and Goldberg [13 was adopted to the sparse graph considered here.

We also employed another network optimization algorithm, namely, the minimum-cost perfect matching problem [11]. We preform a direct calculation based on the dimer representation. As discussed above, this matching problem is that of finding a set of dimers that cover the lattice with minimum cost. The ground-state problem of the TISOS model is precisely of this type when formulated in terms of dimer coverings since such coverings are perfect matchings on the hexagonal lattice. Moreover, unlike the 3-dimensional graphs needed in flow algorithm, the graph here remains planar and much larger systems can be studied with the same computer resources; this allows us to more clearly distinguish $\ln ^{2}(L)$ from $\ln (L)$ roughness in the TISOS model than the min-cut algorithm. Standard algorithms [11,?] are sufficient to solve the problem for smaller systems. We have developed a heuristic algorithm, making use of routines from the $\mathrm{C}++$ LEDA library [14] to implement the minimum-cost dimer matching algorithm. The final sequential code, running on a single IBM RS/6000-390 workstation, takes about 1, 15 and 25 minutes to find the exact groundstate for system sizes of $L=192,420$, and 540 respectively for one instance of disorder realization. Details of the algorithm's implementation will be presented elsewhere [15].

The interface widths, configurational energy, and structure factor were calculated for the 
ground states for samples of various sizes. These quantities were then averaged over at least 2000 samples of each size. The largest length scales for which these many samples were studied were $L=200,420$ for the DS and TISOS models, respectively. The sample-averaged results of our numerical calculations are summarized in Fig. 2 and Fig. 3.

Fig. 2 shows the averaged squared sample widths $\overline{W^{2}}=\overline{\left\{h^{2}\right\}-\{h\}^{2}}$ as a function of sample size. The $\overline{W^{2}}(L)$ curve is not fit well by a straight line on this logarithmic-linear scale: for both the DS and TISOS models, such a fit gives a line well outside the statistical error bars for any range of data over more than a factor of 2 in $L$. This is evidence that the proposal that $\overline{W^{2}}(L) \sim \ln (L)$ does not correctly describe the ground state. The data is however fit quite well by the form

$$
\overline{W^{2}}(L)=C+A \ln (L)+B \ln ^{2}(L),
$$

with the constants being given by $C=0.022,0.39, A=0.023,0.34$, and $B=0.0064,0.060$, for the DS and TISOS models, respectively. The fact that these coefficients satisfy $A=$ $2(B C)^{1 / 2}$ to within a $10 \%$ error indicates that the width $\bar{W}$ is well fit by the form $\bar{W}=$ $C^{1 / 2}+B^{1 / 2} \ln (L)$ even for $L \approx 10$.

We find that even stronger evidence for the super-rough phase with $\overline{W^{2}}(L) \sim \ln ^{2}(L)$ is found by examining the sample-averaged structure factors $\overline{S(k)}$. These structure factors must of course be consistent with the $\overline{W^{2}}$ data, but the shape of these curves for different $L$ shows that the structure factor data have converged well for the sample sizes we examine, so that the curvature of the $\overline{W^{2}}$ plots on a logarithmic scale is not an artifact of small system sizes. Plots of $k^{2} \overline{S(k)}$ are shown in Fig. 3 for the DS and TISOS models. The structure factors are found to be isotropic for small $k$; this is indicated by the convergence of $\overline{S(k)}$ to a single value for all $\vec{k}$ with the same magnitude $k$ as $k \rightarrow 0$. If the coefficient $B$ in Eq. (3) were zero, $k^{2} \overline{S(k)}$ would approach a constant at small $k$. Such convergence is clearly seen, for example, in work on the TISOS model without disorder [16]. For the disordered model, the data does not converge to a constant over the lengths we examined; it is instead fit very well by a logarithmic divergence. The analysis of the structure factor in this fashion allows 
us to clearly distinguish between logarithmically rough and super-rough, since the difference is singular as $k \rightarrow 0$; in the $\overline{W^{2}}(L)$ plots, the difference between the two predictions is additive. The logarithmic divergence is precisely what one expects if $\overline{W^{2}}(L) \sim \ln ^{2}(L)$. This is very strong evidence that $B \neq 0$ in Eq. (3). The prediction of a super-rough ground state is consistent with our data, while the prediction of a logarithmically rough phase is inconsistent with the calculated structure factor.

We also calculated the ground state energy for the fluxlines derived from the TISOSmodel ground states. We find that the fluctuations in the energy per flux line, averaged over samples, $\overline{\Delta E}$ scale as $\overline{\Delta E} \sim L^{0.50 \pm 0.04}$.

In conclusion, we use a maximum-flow algorithm and a minimum-cost perfect matching algorithm to find ground state configurations in the disordered substrate and disordered TISOS models (which is equivalent to finding ground states of many flux lines in a random potential). We find that the ground-state configurations have a $\ln ^{2}(L)$ roughness in both models, in agreement with the renormalization group calculations and in disagreement with the predictions of the lowest-order variational approach. Our results are much clearer than previous numerical work at finite temperature [3,6, 6,7, since the algorithms quickly find exact ground states, so that we can average over many realizations of larger systems and we also study the models far from $T_{g}$, so that the coefficient of the $\ln ^{2}(L)$ is larger. Although the results found here strictly apply to $T=0$ their agreement with these obtained by RG just below $T_{g}$ strongly suggests that the $\ln ^{2}(L)$ behavior holds in the whole $T \leq T_{g}$ glassy phase. These algorithms can be directly extended to study flux lines in many different types of disorder, including columnar and splay defects.

We thank M. Kardar and C. Henley for useful discussions. C. Zeng received support from NSF grants NMR-9217284 and NMR-9419257. A. A. Middleton gratefully acknowledges the Alfred P. Sloan Foundation for support. 


\section{REFERENCES}

[1] J.L. Cardy and S. Ostlund, Phys. Rev. B 25, 6899 (1982); J. Toner and D. P. DiVincenzo, Phys. Rev. B 41, 632 (1990); Y.-C. Tsai and Y. Shapir, Phys. Rev. Lett. 69, $1773(1992)$.

[2] S. E. Korshunov, Phys. Rev. B 48, 3969 (1993); T. Giamarchi and P. Le Doussal, Phys. Rev. Lett. 71, 1530 (1994).

[3] G. G. Batrouni and T. Hwa, Phys. Rev. Lett. 72, 4133 (1994).

[4] H. Rieger, Phys. Rev. Lett. 74, 4964 (1995).

[5] D. J. Lancaster and J. J. Ruiz-Lorenzo, J. Phys. A 28, L577 (1995).

[6] D. Cule and Y. Shapir, Phys. Rev. Lett. 74, 114 (1995).

[7] E. Marinari, R. Monasson, and J. J. Ruiz-Lorenzo, J. Phys. A 28, 3975 (1995).

[8] B. Nienhuis, H J Hilhorst, and H W J Blöte, J. Phys. A: Math. Gen. 17, 3559-2581 (1984).

[9] H W J Blöte, and H J Hilhorst, J. Phys. A: Math. Gen. 15, L631 (1982).

[10] G. H. Wannier, Phys. Rev. 79, 357 (1950).

[11] U. Derigs, Programming in Networks and Graphs, Springer-Verlag, New York (1988).

[12] A. A. Middleton, Phys. Rev. E. 52, R3337 (1995).

[13] Cherkassky and Goldberg, Stanford Technical Report CS-TR-94-1523 (1994).

[14] S. Näher and C. Uhrig, The LEDA User Manual, Verion R 3.3, Martin-Luther Universität Halle-Wittenberg, Germany (1996).

[15] C. Zeng and A. A. Middleton, unpublished.

[16] C. Zeng and C. L. Henley, to be published. 


\section{FIGURES}

FIG. 1. Various representations of the TISOS model. The height variables defined on each vertex of the triangular lattice (dashed lines) are shown in the figure. The equivalent dimer covering is indicated by the thick bonds on the dual hexagonal lattice. The corresponding two fluxlines are displayed as contiguous triple lines. The linear size of the system is denoted by L (=3). Periodic boundary conditions are imposed (twisted from top to bottom).

FIG. 2. Sample-averaged interface widths for the DS and TISOS models, as a function of system size $L$, on a logarithmic scale. The DS square widths $\overline{W^{2}}$ have been multiplied by a scale factor of 10 for comparison with the TISOS results. The fractional statistical errors in $\overline{W^{2}}$ are less than $0.5 \%$; this error is about half of the size of the symbols in the plot. The lines are quadratic fits to all of the data shown; residual errors are within twice the statistical uncertainty for all points.

FIG. 3. Sample averaged structure factors for the DS and TISOS models. The symbols indicate which model and the sample sizes. $\overline{S(k)}$ has been multiplied by $k^{2}$ for clearer comparison with the theoretical predictions, and the curves are normalized so that $k^{2} \overline{S(k)}=1.0$ at $|k|=1$. The error bars shown are greater than the statistical error and reflect the possible error introduced by the smoothing of the $k^{2} \overline{S(k)}$ data over a factor of 2 in $k^{2}$. As $k^{2} \rightarrow 0, k^{2} \overline{S(k)}$ for a logarithmically

rough interface would approach a constant; the apparent logarithmic divergence of $k^{2} \overline{S(k)}$ indicates that the interface is super-rough for the ground states of these two models. Note that the finite-size effects are distinct for the two models: in the DS model, $k^{2} \overline{S(k)}$ rises above a logarithmic form for $k$ near $2 \pi / L$ while in the TISOS model, $k^{2} \overline{S(k)}$ drops below the logarithmic form for $k$ near the finite-volume limit. 
Fig. 1 - Zeng, Middleton, Shapir, "Ground-State Roughness..."

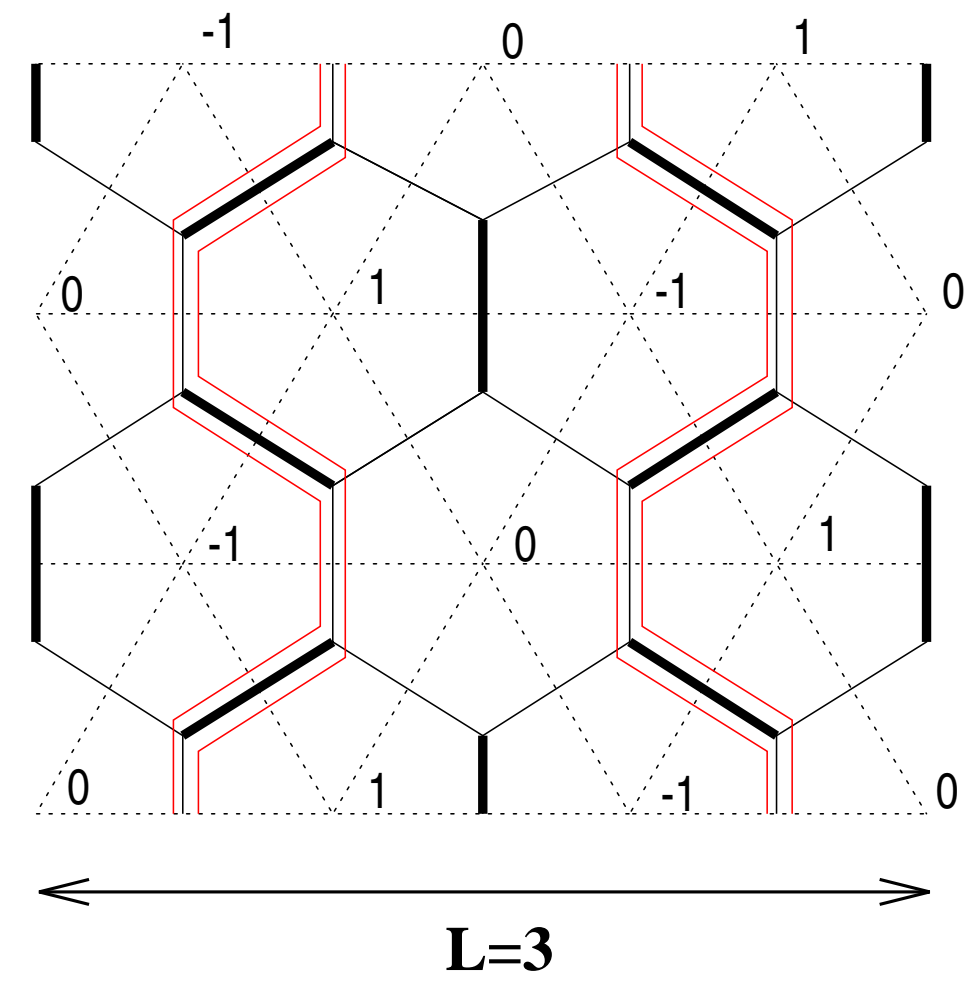


Fig. 2 - Zeng, Middleton, Shapir, "Ground-State Roughness..."

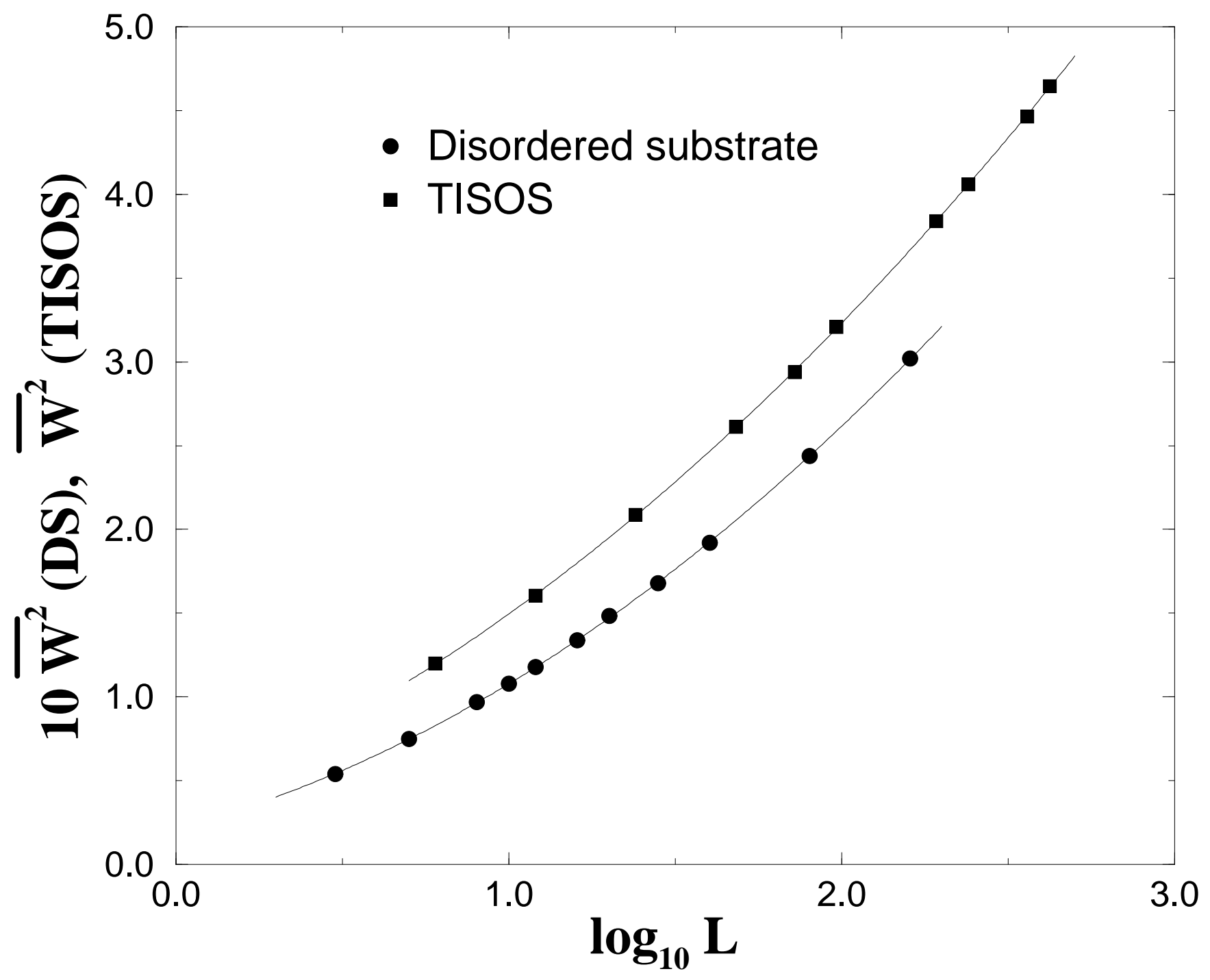


Fig. 3 - Zeng, Middleton, Shapir, "Ground-State Roughness..."

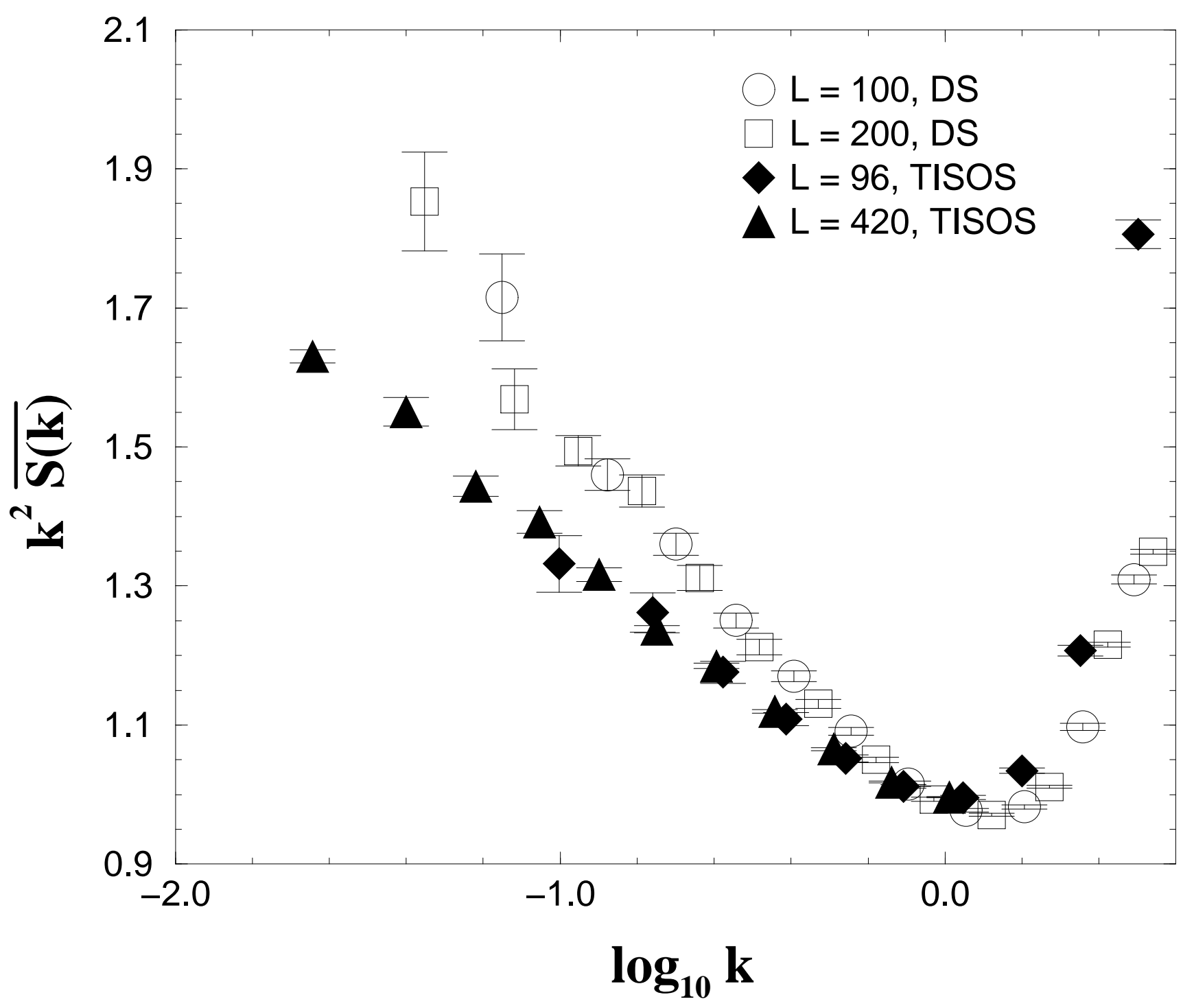

